\title{
LISTA DE LOS COLEÓPTEROS ACUÁTICOS \\ (COLEOPTERA: ADEPHAGA, POLYPHAGA) DE JESÚS MENÉNDEZ, LAS TUNAS, CUBA
}

\author{
Denis Leyva Escobar ${ }^{1}$, Yoandri S. Megna ${ }^{1}$ y Albert Deler-Hernández ${ }^{2}$ \\ ${ }^{1}$ Departamento de Biología, Universidad de Oriente. Patricio Lumumba s/n, Santiago, \\ Santiago de Cuba, Cuba. denis.leyva@cnt.uo.edu.cu; ysuarez@cnt.uo.edu.cu \\ ${ }^{2}$ Department of Zoology, Faculty of Science, Charles University in Prague, Viničná 7, CZ-128 44, \\ Praha 2, Czech Republic
}

\section{RESUMEN}

Se realizó un estudio de la fauna de escarabajos acuáticos del municipio Jesús Menéndez en la provincia de Las Tunas. Fueron registrados 666 ejemplares, pertenecientes a seis familias, 22 géneros y 30 especies. La especie Tropisternus chalybeus Castelnau, 1840 constituye nuevo registro para la región Oriental de Cuba.

Palabras clave: Coleóptera, distribución, nuevo reporte, Cuba.

Title: Checklist of the water beetles (Coleoptera: Adephaga, Polyphaga) from Jesús Menéndez, Las Tunas, Cuba.

\section{ABSTRACT}

A study of the fauna of aquatic beetles from municipality Jesús Menéndez, province Las Tunas is presently being carried out. It was reported 30 species by first time for the area, which distributed in 22 genera and six families. Tropisternus chalybeus Castelnau, 1840 is a new record for Oriental region from Cuba.

Keywords: Coleoptera, distribution, new records, Cuba.

\section{INTRODUCCIÓN}

Los coleópteros acuáticos son uno de los componentes más importantes de los ecosistemas dulceacuícolas (Epler, 2010). Sin embargo, a pesar de su utilidad para determinar el grado de conservación de los hábitats (Sánchez-Fernández et al., 2004) y para regular las poblaciones de mosquitos en condiciones naturales (Lundkvist et al., 2002), los mismos están insuficientemente estudiados en la región Neotropical.

De los cuatro subórdenes en que se divide el orden Coleoptera, solamente tres tienen representantes acuáticos: Myxophaga, Adephaga y Polyphaga (Whiteman y Sites, 2003). En Cuba, son el grupo mayoritario, con 87 familias y 2673 especies (Peck, 2005), pero igual que los demás órdenes de insectos necesita ser mejor estudiado. En recientes estudios se han registrado y descrito nuevas especies para la fauna cubana: Toledo et al. (2011); Megna et al. (2011); Megna y Epler (2012); Deler-Hernández y Delgado (2012).

La provincia de Las Tunas es una de las áreas con reducida o ninguna representación dentro del Sistema Nacional de Áreas Protegidas de Cuba (CNAP, 2004). Lo anterior responde a la antropización, siendo una zona de pastos y cultivos casi en su totalidad. Esto no ha motivado 
campañas de recolectas de los especialista en esta localidad. Muestra de ello es que el principal estudio del siglo XIX sobre coleópteros acuáticos en Cuba, la expedición cubano-rumana, no registró especies para el área. Sin embargo, estudios recientes permitieron describir a Copelatus cordovai Megna y Epler, como una nueva especie de la familia Dytiscidae (Megna y Epler, 2012). También, en los municipios de Amancio y Las Tunas fueron reportadas 42 especies de coleópteros acuáticos (Megna et al., 2011; Deler-Hernández y Delgado, 2012; Megna y Epler, 2012). Estos antecedentes muestran la necesidad de profundizar en la composición faunística de los ecosistemas con abundantes gramíneas y muy deforestados. En este sentido, como las estrategias de conservación de la diversidad biológica deben centrarse en las especies, es necesario recopilar toda la información sobre la fauna de cada ecosistema.

\section{OBJETIVO}

- Realizar un inventario de los coleópteros acuáticos del municipio Jesús Menéndez, provincia Las Tunas, Cuba, aportando algunos datos sobre la biología de las especies.

\section{MATERIALES Y MÉTODOS}

Descripción del área de estudio y localidades de muestreos. El municipio Jesús Menéndez limita por el norte con el Océano Atlántico, al oeste con el municipio Puerto Padre, al este con Velazco, provincia Holguín y por el sur con Majibacoa. Posee una extensión superficial de $638.3 \mathrm{Km}^{2}$. En este municipio predominan las llanuras y los suelos pardos, ferralíticos y hidromórficos. Las temperaturas oscilan alrededor de $\operatorname{los} 26^{\circ} \mathrm{C}$, siendo ligeramente superior en áreas de la costa norte, el mes más cálido es agosto, con temperaturas promedios sobre los $28^{\circ} \mathrm{C}$ y el más fresco es enero. La zona norte es la más seca, asociándose esta condición a la marcada influencia anticiclónica y al régimen de brisas predominantes. Los muestreos se realizaron entre octubre de 2011 y abril de 2013 en el municipio Jesús Menéndez, provincia Las Tunas. Fueron seleccionadas

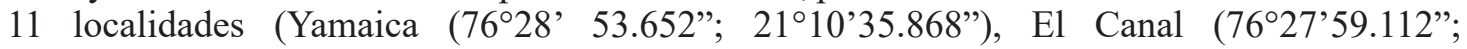

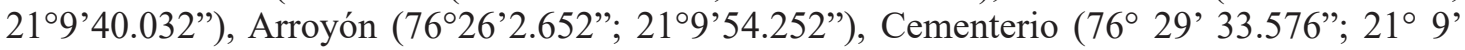

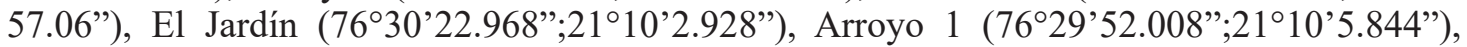

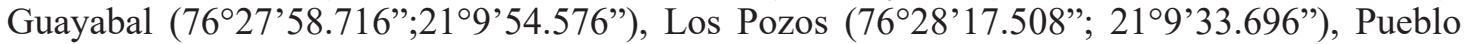
Viejo (76 28'42.123"; $\left.21^{\circ} 9^{\prime} 44.568^{\prime \prime}\right)$, La Lechería (76²8'25.392"; $21^{\circ} 8^{\prime}$ '59.172") y Herradura (76²6'42.72"; $21^{\circ} 9$ ' 26.544"), Fig.1.

Método de recolecta. Para las recolecta se empleó el jameo acuático, el cual se realizó con una red triangular de $30 \mathrm{~cm}$ en cada lado y $45 \mathrm{~cm}$ de fondo, con ojo de malla inferior a 0,05 $\mathrm{mm}$. Se emplearon las recolectas en los hábitats lénticos y lóticos por tiempo de 2 horas, abarcando todos los microhábitats ecológica y fisionómicamente diferentes. Todos los ejemplares (adultos) colectados fueron conservados en alcohol al 70\%, y etiquetados para su posterior identificación en el Laboratorio de Entomología del Departamento de Biología de la Universidad de Oriente. Posteriormente fueron depositados en la colección del Museo de Historia Natural Charles T. Ramsden de la Torre (CZCTR) y en la colección Zoológica del Departamento de Zoología (BSC-E) del Centro Oriental de Ecosistemas y Biodiversidad (BIOECO), Santiago de Cuba, Cuba.

Taxonomía. Para la identificación del material se utilizaron diferentes claves según el nivel taxonómico (Epler, 1996, 2010; Larson et al., 2000; Megna et al., 2011; Megna y Epler, 2012; Deler-Hernández y Delgado, 2012). 


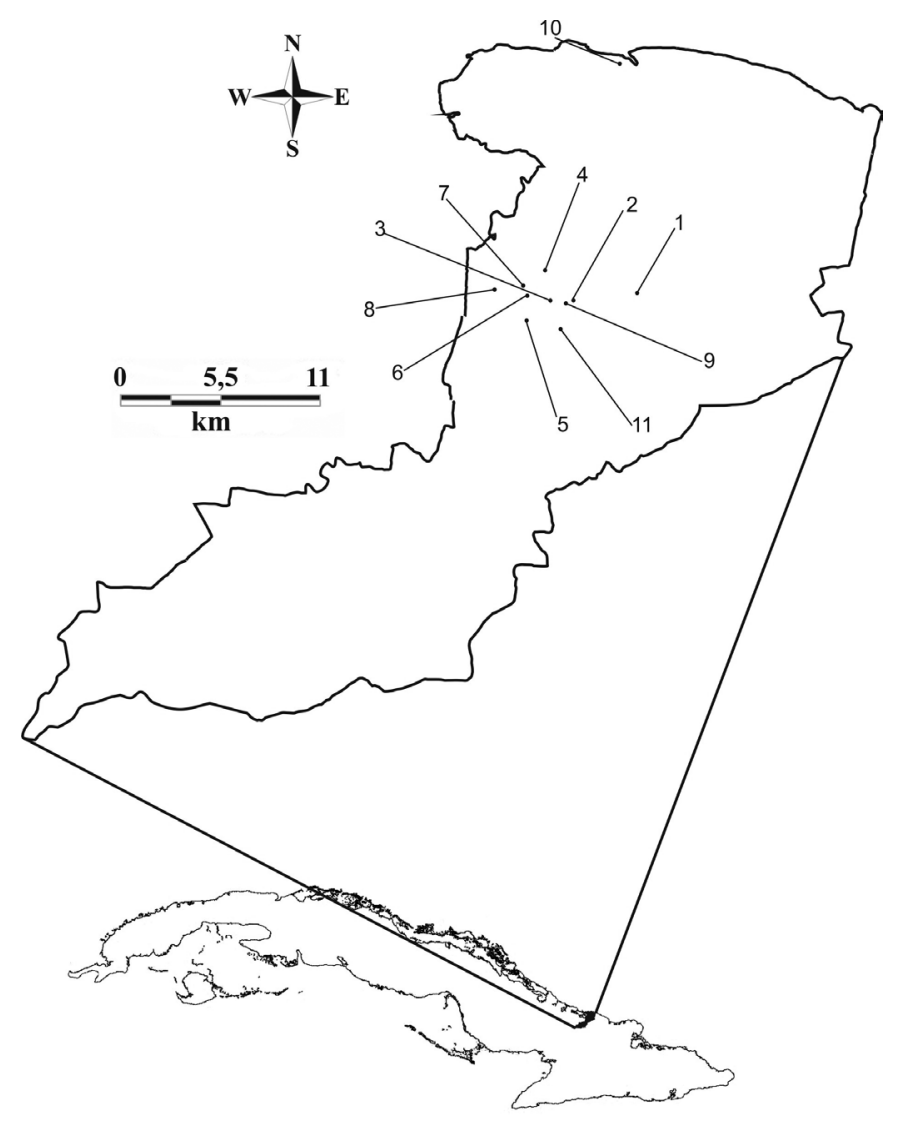

Figura 1. Localidades de recolectas de especies de coleópteros acuáticos en el municipio Jesús Menéndez, provincia Las Tunas, Cuba. 1) Yamaica, 2) El Canal, 3) Arroyón, 4) Cementerio, 5) El Jardín, 6) Arroyo 1, 7) Guayabal, 8) los Pozos, 9) Pueblo Viejo, 10) Herradura, 11) La Lechería.

\section{RESULTADOS}

\section{LISTA ANOTADA DE LAS ESPECIES DE ADEPHAGA Y POLYPHAGA DE JESÚS MENÉNDEZ, LAS TUNAS, CUBA.}

Se recolectaron 666 ejemplares de Adephaga y Polyphaga para el municipio Jesús Menéndez, correspondiendo a seis familias, 9 subfamilias y 30 especies. Todas las especies constituyen nuevos registros para la localidad. El mayor número de ejemplares corresponde a las familias Dytiscidae con 357 e Hydrophilidae con 82.

* = Nuevo registro de especie para la región Oriental.

Orden Coleoptera

Familia Dytiscidae

Subfamilia Laccophilinae

Género Laccophilus 


\section{L. proximus (Say, 1823)}

Material examinado. Cuba. Las Tunas. Pueblo Viejo, 1-III-2013, D. Leyva col; 8. La Turbina, 1-III-2013, D. Leyva col; 16. Yamaica, 1-III-2013, D. Leyva col; 6. El Canal, 15-III-2013, D. Leyva col; 6. Arroyón, 15-III-2013, D. Leyva col; 1. El Canal, 30-III-2013, D. Leyva col; 6. Yamaica, 29-III-2013, D. Leyva col; 6. El Guayabal, 30-III-2013, D. Leyva col; 2. Arroyo 1, 29-III-2013, D. Leyva col; 2. Laguna temporal, 19-X-2011, D. Leyva col; 2. Arroyo 1, 12-IV2013, D. Leyva y Y. S. Megna col; 22. Herradura, 13-IV-2013, D. Leyva y Y. S. Megna col; 4. El Jardín, 12-IV-2013, D. Leyva y Y. S. Megna col; 2. Cementerio, 17-IV-2013, D. Leyva col; 17. Laguna de la Lechería, 15-IV-2013, D. Leyva col; 75. Yamaica, 12-IV-2013, D. Leyva y Y. S. Megna col; 8. El Canal, 12-IV-2013, D. Leyva y Y. S. Megna col; 7. El Guayabal, 12-IV-2013, D. Leyva y Y. S. Megna col; 4.

Biología. L. proximus fue registrada en todas las localidades de recolecta. Es una de las especies más abundantes de la familia Dytiscidae y es considerada como una de las que tienen más amplia distribución en Cuba (Megna et al., 2011).

L. vacaensis vacaensis (Young, 1953)

Material examinado. Cuba. Las Tunas. El Canal, 30-III-2013, D. Leyva col; 2. Arroyo 1, 29-III2013, D. Leyva col; 1. Yamaica, 29-III-2013, D. Leyva col; 2. La Turbina, 1-III-2013, D. Leyva col; 1. El Canal, 12-IV-2013, D. Leyva y Y.S. Megna col; 2.

Biología. L. vacaensis vacaensis fue recolectada en hábitats lénticos permanentes y temporales de elevadas temperaturas y con fondo fangoso o arenoso-fangoso, coincidiendo con lo propuestos para las especies de Laccophilus por Megna et al. (2011).

\section{Subfamilia Dytiscinae \\ Género Thermonectus}

T. circumscriptus (Latreille, 1809)

Material examinado. Cuba. Las Tunas. Pueblo Viejo, 1-III-2013, D. Leyva col; 2. El Canal, 15III-2013, D. Leyva col; 3. El Guayabal, 30-III-2013, D. Leyva col; 1. Laguna de la Lechería, 15-IV-2013, D. Leyva col; 1. Herradura, 13-IV-2013, D. Leyva y Y.S. Megna col; 2.

Biología. T. circumscriptus fue recolectada en la gran mayoría de las localidades lénticas y lóticas del área de estudio. Es junto a T. basillaris basillaris la más abundante del género, lo cual coincide con lo planteado por Alarie et al. (2009).

\section{T. basillaris basillaris (Harris, 1829)}

Material examinado. Cuba. Las Tunas. El Canal, 15-III-2013, D. Leyva col; 5. La Turbina, 19-X-2011, D. Leyva col; 3. Laguna de la Lechería, 15-IV-2013, D. Leyva col; 3. Arroyo 1, $12-$ IV-2013, D. Leyva y Y. S. Megna col; 1. Herradura, 13-IV-2013, D. Leyva y Y. S. Megna col; 5. El Canal, 12-IV-2013, D. Leyva y Y. S. Megna col; 5. El Canal, 12-IV-2013, D. Leyva y Y. S. Megna col; 2.

Biología. T. basillaris fue recolectada en hábitats lénticos permanentes y temporales con fondo fangoso, fangoso-pedregoso, ubicados en zonas de baja altitud. También ha sido recolectada en lagunas costeras salobres. 


\title{
Género Prodaticus
}

P. rimosus (Aubé, 1838)

Material examinado. Cuba. Las Tunas. El Canal, 15-III-2013, D. Leyva col; 1.

Biología. P. rimosus fue recolectada en hábitats lénticos permanentes con fondo fangoso y con presencia de vegetación.

\author{
Subfamilia Copelatinae \\ Género Copelatus
}

C. cordovai (Megna y Epler, 2012)

Material examinado. Cuba. Arroyo 1, 29-III-2013, D. Leyva col; 1.Yamaica, 12-IV-2013, D. Leyva y Y. S. Megna col; 3.Arroyo 1, 12-IV-2013, D. Leyva y Y. S. Megna col; 2. El Guayabal, 30-III-2013, D. Leyva col; 2. El Jardín, 12-IV-2013, D. Leyva y Y. S. Megna col; 1. El Canal, 30-III-2013, D. Leyva col; 1. Laguna de la Lechería, 15-IV-2013, D. Leyva col; 3. Cementerio, 17-IV-2013, D. Leyva col; 2.

Biología. Esta especie fue recolecta en lagunas permanentes en áreas muy antropizadas. Previo a este estudio, C. cordovai había sido registrada en hábitats lénticos ubicados en zonas bajas de área de cultivos en Amancio, Las Tunas y Cauto Cristo, Granma (Megna y Epler, 2012).

\section{Subfamilia Hydroporinae \\ Género Bidessonotus}

\section{B. browneanus (Balfour-Browne, 1947)}

Material examinado. Cuba. Las Tunas. Yamaica, 1-III-2013, D. Leyva col; 1. El Canal, 15III-2013, D. Leyva col; 1. Arroyón, 15-III-2013, D. Leyva col; 1.Yamaica, 29-III-2013, D. Leyva col; 83. El Jardín, 29-III-2013, D. Leyva col; 2. El Guayabal, 30-III-2013, D. Leyva col; 1.Arroyo 1, 12-IV-2013, D. Leyva y Y. S. Megna col; 11. El Jardín, 12-IV-2013, D. Leyva y Y. S. Megna col; 41. El Guayabal, 12-IV-2013, D. Leyva y Y. S. Megna col; 4. Laguna de la Lechería, 15-IV-2013, D. Leyva col; 1. Cementerio, 17-IV-2013, D. Leyva col; 8. Yamaica, 12IV-2013, D. Leyva y Y. S. Megna col; 80. Herradura, 13-IV-2013, D. Leyva y Y. S. Megna col; 8.

Biología. B. browneanus fue la que aportó el mayor número de ejemplares, recolectados en localidades con fondo fangoso, aguas turbias y abundante vegetación acuática.

\section{Género Anodocheilus}

\section{A. exiguus (Aubé, 1838)}

Material examinado. Cuba. Las Tunas. Yamaica, 29-III-2013, D. Leyva col; 1.Arroyo 1, 12IV-2013, D. Leyva y Y. S. Megna col; 1. El Jardín, 12-IV-2013, D. Leyva y Y. S. Megna col; 2. Yamaica, 12-IV-2013, D. Leyva y Y. S. Megna col; 1.

Biología. A. exiguus fue recolectada en los márgenes de lagunas y charcas, así como en remansos de pequeños arroyos localizados en zonas de baja altitud. Generalmente fue encontrada en lugares con fondo fangoso, aguas turbias y abundante vegetación acuática. 


\section{Género Hydrovatus}

H. caraibus (Sharp, 1882)

Material examinado. Cuba. Las Tunas. Arroyo 1, 29-III-2013, D. Leyva col; 1. Yamaica, 29III-2013, D. Leyva col; 1. Yamaica, 12-IV-2013, D. Leyva y Y. S. Megna col; 2. Laguna de la Lechería, 15-IV-2013, D. Leyva col; 1. El Jardín, 12-IV-2013, D. Leyva y Y. S. Megna col; 1. Cementerio, 17-IV-2013, D. Leyva col; 1.

Biología. H. caraibus fue recolectada en hábitats lénticos temporales y permanentes, con aguas turbias y fondo fangoso.

\section{Género Liodessus}

L. noviaffinis (Miller, 1998)

Material examinado. Cuba. Las Tunas. Yamaica, 29-III-2013, D. Leyva col; 1.

Biología. L. noviaffinis fue colectada en el la zona de remanso de un río, con abundante vegetación acuática, flotante y saliente. Además con un fondo fangoso y el agua turbia.

Comentario. Este constituye un segundo registro de L. noviaffinis para Cuba. Spangler (1981) la reportó para Pinares de Mayarí, en la provincia Holguín, con el nombre Liodessus affinis. Sin embargo, Miller (1998) determinó que Liodessus affinis es un grupo conformado por varias especies y asignó el nombre de L. noviaffinis al registro cubano.

\section{Género Pachydrus}

P. obniger (Chevrolat, 1863)

Material examinado. Cuba. Las Tunas. Yamaica, 12-IV-2013, D. Leyva y Y. S. Megna col; 1.

Biología. P. obniger fue recolectada hábitats lóticos, con aguas turbias y fondo fangoso y abundante vegetación acuática, coincidiendo con lo propuesto por Megna y Deler-Hernández (2006).

\section{Género Celina}

\section{C. slossoni (Mutchle, 1918)}

Material examinado. Cuba. Las Tunas. Yamaica, 12-IV-2013, D. Leyva y Y. S. Megna col; 1.

Biología. C. slossoni fue colectada en hábitats lénticos permanentes localizados en zonas de baja altitud. Esta especie estuvo asociada a fondos fangosos y ambientes con abundante vegetación acuática.

Familia Hydrophilidae

Subfamilia Hydrophilinae

Género Tropisternus

T. collaris (Fabricius, 1775)

Material examinado. Cuba. Las Tunas. Pueblo Viejo, 1-III-2013, D. Leyva col; 4.

Biología. T. collaris fue reportado para una sola localidad en hábitats lóticos, con agua corriente 
y fondo pedregoso. Previo a este estudio, la especie había sido registrada en un arroyo y una laguna permanente de la provincia Santiago de Cuba (Spangler, 1981, Deler-Hernández y CalaRiquelme, 2010).

\section{T. lateralis (Fabricius, 1775)}

Material examinado. Cuba. Las Tunas. La Turbina, 1-III-2013, D. Leyva col; 6. Cementerio, 17-IV-2013, D. Leyva col; 2.Laguna de la Lechería, 15-IV-2013, D. Leyva col; 1.Herradura, 13-IV-2013, D. Leyva y Y. S. Megna col; 2. E1 Jardín, 12-IV-2013, D. Leyva y Y. S. Megna col; 1. El Canal, 12-IV-2013, D. Leyva y Y. S. Megna col; 4.

Biología. T. lateralis se reportó asociada a lagunas permanentes con fondo cubierto de fango, poca profundidad y abundante vegetación de orilla.

\section{T. chalybeus (Castelnau, 1840)*}

Material examinado. Cuba. Las Tunas. Arroyo 1, 29-III-2013, D. Leyva col; 1. El Canal, 30-III2013, D. Leyva col; 1.

Biología. T. chalybeus fue registrada en hábitats lenticos y lóticos de baja altitud. La especie había sido registrada en Cárdenas, provincia Matanzas. Este constituye el primer reporte de esta especie para la región Oriental.

T. mergus (Say, 1835)

Material examinado. Cuba. Las Tunas. El Canal, 30-III-2013, D. Leyva col; 6.

Biología. T. mergus fue registrada en un hábitat lótico con fondo fangoso y agua turbia.

\section{Género Berosus}

B. undatus (Fabricius, 1792)

Material examinado. Cuba. El Canal, 15-III-2013, D. Leyva col; 1. Laguna de la Lechería, 15IV-2013, D. Leyva col; 28. Herradura, 13-IV-2013, D. Leyva y Y. S. Megna col; 9.

Biología. B. undatus fue recolectada en lagunas interiores y costeras con fondo fangoso, coincidiendo con lo propuesto para las especies de Berosus por Deler-Hernández et al. (2013).

B. interstitialis (Knisch, 1924)

Material examinado. Cuba. Las Tunas. Laguna de la Lechería, 15-IV-2013, D. Leyva col; 1.

Biología. B. interstitialis fue recolectada en una laguna permanente con fondo fangoso.

\section{Género Paracymus}

\section{P. lodingi (Fall, 1910)}

Material examinado. Cuba. Las Tunas. El Jardín, 29-III-2013, D. Leyva col; 2.Cementerio, 17IV-2013, D. Leyva col; 6.

Biología. P. lodingi fue recolectada en hábitats lénticos de fondo fangoso. Spangler (1981) reportó esta especie para el occidente de Cuba, incluyendo la Isla de la Juventud. 


\section{Subfamilia Enochrinae \\ Género Enochrus}

E. pygmaeus (Fabricius, 1792)

Material examinado. Cuba. Las Tunas. Yamaica, 1-III-2013, D. Leyva col; 1.E1 Jardín, 29-III2013, D. Leyva col; 2. Cementerio, 17-IV-2013, D. Leyva col; 5.

Biología. E. pygmaeus fue recolecta en hábitats lénticos y lóticos con fondo fangoso. Previo a este estudio la especie en Cuba siempre había sido reportada para ambientes lóticos (Spangler, 1981).

Familia Hydraenidae

Subfamilia Hydraeninae

Género Hydraena

H. decui (Spangler, 1980)

Material examinado: Cuba. Las Tunas. Rio Arroyón, 15-III-2013, D. Leyva col; 2.

Biología. H. decui fue recolectada en hábitat léntico de baja altitud. Sin embargo, DelerHernández y Delgado (2012) determinaron que esta especie es típica de arroyos en zonas montañosas.

H. guadelupensis (Orchymont, 1923)

Material examinado: Cuba. Las Tunas. Yamaica, 29-III-2013, D. Leyva col; 2.

Biología: H. guadelupensis fue recolectada en hábitat léntico de baja altitud. Sin embargo, Deler-Hernández y Delgado (2012) determinaron que esta especie es típica de arroyos en zonas montañosas.

Subfamilia Ochthebiinae

Género Gymnochthebius

G. fossatus (LeConte, 1855)

Material examinado. Cuba. Las Tunas. Arroyo 1, 29-III-2013, D. Leyva col; 11. Yamaica, 29III-2013, D. Leyva col; 4. Yamaica, 12-IV-2013, D. Leyva y Y. S. Megna col; 24. Cementerio, 17-IV-2013, D. Leyva col; 12.

Biología. G. fossatus fue recolectada en hábitats tanto lénticos como y lóticos de baja altitud. Lo anterior coincide con los propuesto para especie por Deler-Hernández y Delgado (2012).

\section{Género Ochthebius}

O. attritus (LeConte, 1878)

Material examinado. Cuba. Las Tunas. Arroyo 1, 29-III-2013, D. Leyva col; 1.

Biología. O. attritus fue recolectada en el remanso de un arroyo y constituyó la especie menos abundante de la familia Hydraenidae en el área de estudio. 


\section{Familia Haliplidae \\ Género Haliplus}

H. havaniensis (Wehncke, 1880)

Material examinado. Cuba. Las Tunas. Yamaica, 1-III-2013, D. Leyva col; 1.

Biología. H. havaniensis fue recolectada en el remanso de un río con abundante vegetación acuática, flotante y saliente. Esta es la especies más común del género, con más amplia distribución (Van Vondel y Spangler, 2008)

Familia Noteridae

Subfamilia Noterinae

Género Hydrocanthus

H. oblongus (Sharp, 1882)

Material examinado. Cuba. Las Tunas. Yamaica, 29-III-2013, D. Leyva col; 1. Cementerio, 17IV-2013, D. Leyva col; 2.

Biología. H. oblongus fue recolectada en el remanso de un río con abundante vegetación acuática, flotante y saliente.

\section{Género Suphisellus}

S. insularris (Sharp, 1882)

Material examinado. Cuba. Las Tunas. Yamaica, 12-IV-2013, D. Leyva y Y. S. Megna col; 1. Cementerio, 17-IV-2013, D. Leyva col; 2.

Biología. S. insularris fue recolectada en el remanso de un río con abundante vegetación acuática, flotante y saliente.

S. nigrinus (Aubé, 1838)

Material examinado. Cuba. Las Tunas. Cementerio, 17-IV-2013, D. Leyva col; 6.

Biología. S. nigrinus fue recolecta en una laguna permanente con fondo fangoso.

\section{Género Notomicrus}

N. sharpi (Balfour-Browne, 1939)

Material examinado. Cuba. Las Tunas. El Jardín, 12-IV-2013, D. Leyva y Y. S. Megna col; 3.

Biología. N. sharpi fue recolecta en una laguna con fondo fangoso y aguas turbias.

Familia Gyrinidae

Subfamilia Gyrininae

Género Dineutus

D. americanus (Linnaeus, 1767)

Material examinado. Cuba. Las Tunas. El Canal, 30-III-2013, D. Leyva col; 2. El Canal, 12-IV- 
2013, D. Leyva y Y. S. Megna col; 2.

Biología. Esta especie fue registrada en una sola localidad y con un reducido número de individuos. D. americanus es una especie de amplia distribución, generalmente asociada con los arroyos de montaña (Peck, 2005), pero también puede estar dispersa en zonas bajas.

\section{AGRADECIMIENTOS}

La realización de este estudio no hubiera sido posible sin el apoyo de David R. Leyva Cabrera, Madelín Escobar Pupo, Ramón F. Leyva Rojas y Coralia Cabrera Tolosa. El estudio fue apoyado por el Grant SVV-2013-267 201 a A. Deler-Hernández.

\section{LITERATURA CITADA}

Alarie, Y., Y. S. Megna y A. Deler-Hernández. 2009. First West Indies records of Thermonectus succinctus (Aubé, 1838), with notes on other Cuban species (Coleoptera: Dytiscidae). Koleopterologische Rundschau 79: 5-16.

Aubé, C. 1838. Hydrocanthares et gyriniens. En: P. F. M. A. Dejean (eds.), Speciesgéneral des coléoptères de la collection de M. le Comte Dejean. Vol. VI. xvi+804 pp. Paris: Francia. Belgique 63: 33-44.

Balfour-Browne, J. 1939. A contribution to the study of the Dytiscidae. - I. (Coleoptera, Adephaga). The Annals and Magazine of Natural History (11) 3: 97-114.

Balfour-Browne, J. 1947. A revision of the genus Bidessonotus Régimbart (Coleoptera: Dytiscidae). Transactions of the Royal Entomological Society 98: 425-448.

Castelnau, F. Laporte de. 1840. Histoire de naturelle des Animaux articulés. Histoire naturelle des Insectes Coléoptéres Vol. 2. (Nécrophages-Triméres). 565 pp., 38 pl. -P. Deménil, Paris.

Chevrolat, L. A. A. 1863. Coléoptère de $1^{e}$ ÎIe de Cuba. Notes, synonymies et descriptions d'espéces nouvelles. Familles des cicindélètes, carabiques, dytiscides, gyrinides et palpicornes. Annales de la Société Entomologique de France 4 (3): 183-210.

CNAP. 2004. Áreas Protegidas de Cuba. Ministerio de Ciencia, Tecnología y Medio Ambiente, La Habana, Cuba, 112 pp.

Deler-Hernández, A. y F. Cala-Riquelme. 2010. Escarabajos acuáticos de la reserva florística manejada "Monte Barranca", Santiago de Cuba, Cuba (Coleoptera: Adephaga, Polyphaga). Novitates Caribaea 3: 69-76.

Deler-Hernández, A y J. A. Delgado, 2012. The Hydraenidae of Cuba (Insecta: Coleoptera) I: Contribution to the fauna of eastern Cuba. Zootaxa 3478: 213-238.

Epler J. H. 1996. Identification manual for the water beetles of Florida (Coleoptera: Dryopidae, Dytiscidae, Elmidae, Gyrinidae, Haliplidae, Hydraenidae, Hydrophilidae, Noteridae, Psephenidae, Ptilodactylidae, Scirtidae). Florida Department of Environmental Protection, Tallahassee, iv $+253 \mathrm{pp}$.

Epler J. H. 2010: The water beetles of Florida - an identification manual for the families Chrysomelidae, Curculionidae, Dryopidae, Elmidae, Gyrinidae, Haliplidae, Helophoridae, 
Hydraenidae, Hydrochidae, Hydrophilidae, Noteridae, Psephenidae, Ptilodactylidae and Scirtidae. Florida Department of Environmental Protection, Tallahassee, $399+$ iv pp.

Fabricius, J. C. 1775. Systema entomologiae, sistens Insectorum classes, ordines, genera, species adiectis synonymis, locis, descriptionibus, observationibus. Flensburgi et Lipsiae: Libraria Korte, xxxii +832 pp.

Fabricius, J. C. 1792. Entomologia systematica emendata et aucta. Vol. I: 1. Hafniae, Francia, 330 pp.

Fall, H. C. 1910. Miscellaneous notes and descriptions of North American Coleoptera. Transactions of the American Entomological Society 36: 89-197.

Harris, T. W. 1829. Corrections and additions for the "Contributions to entomology". New England Farmer 8 (1): 1-2.

Larson, D. J., Y. Alarie y R. E. Roughley. 2000. Predaceous Diving Beetles (Coleoptera: Dytiscidae) of the Nearctic Region, with emphasis on the fauna of Canada and Alaska. Ottawa: NRC Research Press. X +971 pp.

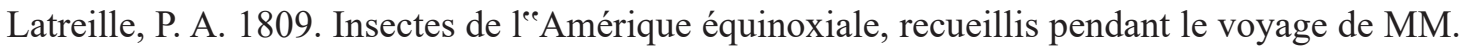
de Humboldt et Bonpland. En: F. H. A. von Humboldt, (eds.), Voyage de Humboldt et Bonpland. 2me partie. Recueil d'observations de zoologie et d'anatomie comparée. Tom 1. Paris, Francia, 412 pp., 26 pls.

LeConte, J. L. 1855. Synopsis of the Hydrophilidae of the United States. Proceeding of the Academy of Natural Sciences of Philadelphia 7: 356-375.

LeConte, J. L. 1878. Additional descriptions of the new species. Proceedings of the American Philosophical Society 17: 373-434.

Linnaeus, C. 1767. Systema naturae, per regna tria naturae, secundum classes, ordines, genera, species, cum caracteribus, differentiis, synonymis, locis. Vol. 1. Part 2. Editio duodecima, reformata. Holmiae: L. Salvii, pp. 533-1327.

Lundkvist, E., J. Landin y F. Karlsson. 2002. Dispersing diving beetles (Dytiscidae) in agricultural and urban landscapes in south-eastern Sweden. Annales Zoologici Fennici 39: 109-123.

Megna S. Y. y A. Deler. 2006. Hydradephaga (Coleoptera: Adephaga) del Sector Baracoa del Parque Nacional Alejandro de Humboldt, Cuba. Boletín Sociedad Entomológica Aragonesa 38: $153-156$.

Megna, Y. S. y J. H. Epler. 2012. Copelatus Erichson, 1832 (Coleoptera: Dytiscidae: Copelatinae) from Cuba. Acta Entomologica Musei Nationalis Pragae 52 (2): 383-410.

Megna Y. S, A. Deler-Hernández y G. L. Challet. 2011. Description of a new species of Laccophilus Leach (Coleoptera: Dytiscidae), with notes on other Cuban species. The Coleopterists Bulletin 65(3): 213-226.

Miller, K. B. 1998. Revision of the Nearctic Liodessus affinis (Say 1823) species group (Coleoptera: Dytiscidae, Hydroporinae, Bidessini). Entomologica scandinavica 29: 281314. 
Orchymont, A. d'. 1923. Les Hydraena Americaines. Annales de la Société Entomologique de Belgique 63: 33-44.

Peck, S. B. 2005. A checklist of the beetles of Cuba with data on distribution and bionomics (Insecta: Coleoptera). Arthropods of Florida and Neighboring Land Areas 18: 1-241.

Sánchez-Fernández, D., Abellán, P., Velasco, J. y A. Millán. 2004. Áreas prioritarias de conservación en la cuenca del río Segura utilizando los coleópteros acuáticos como indicadores. Limnetica 23(3-4): 209-227.

Say, T. 1823. Descriptions of insects of the families of Carabici and Hydrocanthari of Latreille, inhabiting North America. Transactions of the American Philosophical Society N.S. 2 (1825), (1): 1-109.

Say, T. 1835. Descriptions of the new North American Coleopterous Insects, and observations on some already described. Boston Journal of Natural History 1 (1835-37): 151-203.

Sharp, D. 1882. On aquatic carnivorous Coleoptera or Dytiscidae. Scientific Transactions of the Royal Dublin Society 2 (2): 179-1003.

Spangler, P. J. 1980. Two new species of aquatic beetles of the genus Hydraena from Cuba (Coleoptera: Hydraenidae). Proceedings of the Entomological Society of Washington 82: 329-333.

Spangler, P. J. 1981. Suplement to the aquatic and semiaquatic Coleoptera of Cuba collected by the Biospeleological Expeditions to Cuba by the Academies of Science of Cuba and Romania. En: Résultats des Expéditions Biospéologiques Cubano-Roumaines à Cuba, 3. pp. 145-171. Editura Academiei Republicii Socialiste România, Gutenberg, România.

Toledo, M., Y. S. Megna y Y.Alarie. 2011. Description of a new species of Laccodytes Régimbart, 1895 (Coleoptera, Dytiscidae, Laccophilinae) from Cuba. Zootaxa 2792 63-67.

Van Vondel, B. J. y P. J. Spangler. 2008. Revision of the Haliplidae of the Neotropical Region including Mexico (Coleoptera: Haliplidae). Koleopterologische Rundschau 78: 69-194.

Wehncke, E. 1880. Neue Haliplus. Stettiner Entomologische Zeitung 41: 72-75.

Whiteman, N. K. y R. Sites. 2003. Lentic beetles of the Missouri prairie region: habitat and regional associations, with keys to the Hydradephaga. Transactions of the American Entomological Society 129: 185-243.

Young, F.N. 1953. A new Laccophilus from Florida, with notes on other species of the genus (Coleoptera: Dytiscidae). The Florida Entomologist 36: 31-34.

[Recibido: 08 de enero, 2014. Aceptado para publicación: 07 de julio, 2014] 